\title{
Dois anos de pandemia: um balanço
}

\author{
| ${ }^{1}$ Mario Roberto Dal Poz |
}

${ }^{1}$ Instituto de Medicina Social - Universidade do Estado do Rio de Janeiro, Rio de Janeiro-RJ, Brasil (dalpozm@uerj.br) ORCID: 0000-0002-3005-3280.

DOI: http://dx.doi.org/10.1590/S0103-73312021310400

A pandemia pelo novo coronavirus (COVID-19) desvelou a importância inegável do sistema de saúde brasileiro. Entretanto, também destacou suas vulnerabilidades, especialmente a distribuição geográfica desigual dos profissionais de saúde e do acesso da população aos serviços de saúde de média e alta complexidade.

Passados quase dois anos da pandemia, o balanço não é muito animador. Além da tragédia expressa pelos mais de seiscentos mil mortos no Brasil, a pandemia desencadeou ou expôs deficiências em áreas que tinham sido percebidas historicamente como fundamentais do SUS (Sistema Único de Saúde) e da saúde pública brasileira, como a vigilância epidemiológica e a rede de unidades de saúde familiar e de agentes de saúde comunitários. A única exceção parece se encontrar no relativo sucesso da vacinação, descontados a demora, os problemas e conflitos para a aquisição e distribuição das vacinas pelo Governo Federal, através do Ministério da Saúde.

E esta situação não parece ter perspectivas de melhora, dado que a falta de liderança e coordenação federal e a resposta desconexa à pandemia estão relacionadas à crise política e financeira exacerbadas pela pandemia, representando desafios significativos para o futuro do Sistema Único de Saúde. Já existem sinais de um agravamento da crise de saúde, incluindo o agravamento de doenças não transmissíveis e outros problemas de saúde na população e reduçóes substanciais na cobertura vacinal e outras provisões sanitárias básicas. 
O mais grave é a inexistência de testagem em massa da população e de grupos sensíveis, como profissionais de saúde e da educação, impedindo o monitoramento adequado da pandemia e do impacto das medidas de flexibilização das atividades econômicas e mobilidade da população. Uma consequência desse problema é a discussão, com decisôes contraditórias, sobre a permanência ou não das medidas não-farmacológicas para o controle epidemiológico, como o uso de máscaras e o retorno às atividades presenciais.

O Ministério da Saúde não harmonizou esse processo, perdendo credibilidade e capacidade de liderança. Há Estados brigando com outros Estados ou municípios.

Aqui no Rio de Janeiro, como em outros estados, faltou e continua a faltar a articulação entre os diversos entes públicos, tanto nas medidas de controle nãofarmacológicas, como nos esquemas de vacinação.

Essa resposta insuficiente e tardia para o enfrentamento da covid-19 foi surpreendente em funçáo de uma trajetória anterior que demonstrava a capacidade brasileira de acionar políticas e programas efetivos, com relativo sucesso e eficácia no controle de determinados riscos e agravos como HIV/AIDS, tabagismo e, mais recentemente, Zica. Em todos estes casos, ficou demonstrada a potencialidade e o impacto positivo sobre a saúde das populações afetadas, quando à determinação política dos 3 níveis do SUS, governos federal e estadual e municipal, se associava o envolvimento dos cientistas de diferentes disciplinas e o engajamento das comunidades e famílias, numa abordagem coletiva sobre os problemas e suas consequências potencialmente devastadoras.

A omissão de um grande número das sociedades médicas foi decisiva para a disseminação de terapêuticas sem comprovação cientifica, com a notável exceção dos infectologistas e das associações de saúde pública.

Desde o início da pandemia até os dias atuais as tensôes se mantêm e não dão sinais de arrefecimento. Podemos afirmar que o país teria como organizar uma resposta muito mais efetiva, mas isso não ocorreu.

Um aspecto que merece ser destacado é o papel das entidades representativas da sociedade civil, sociedades médicas, instituiçôes acadêmicas e centros de pesquisa brasileiros, especialmente das instituiçôes públicas de pesquisa, que compôem o sistema brasileiro de ciência, tecnologia e inovação. Apesar de enfrentar a crise mais grave de sua história devido ao corte radical de seus recursos financeiros, mas também devido aos ataques sistemáticos originados pelo governo federal, as 
instituiçôes de pesquisa científica e tecnológica no Brasil tem produzido estudos essenciais para compreender o complexo e multifacetado processo da pandemia, além de participarem de projeto, nacionais e internacionais, em busca de produtos que se mostrem seguros e eficazes, com ênfase no desenvolvimento e teste de vacinas.

Docentes, pesquisadores e alunos do campo da saúde coletiva, dentre as quais se inclui o Instituto de Medicina Social, tem desenvolvido diversas iniciativas e atividades desde o início da pandemia pelo novo coronavirus, em 2020, com o fornecimento de informaçôes, estudos, pesquisas, estatísticas, projetos, planos de ação, estratégias, opiniōes técnicas e todos os demais instrumentos e ferramentas, para o enfrentamento das diferentes etapas da pandemia, incluindo sua difusão e comunicação junto à população.

Aproveitamos o último número de 2021 para enfatizar o caráter também coletivo das publicaçóes científicas e agradecer a colaboração dos pareceristas que generosamente contribuíram com seu tempo e sua expertise para viabilizar as ediçôes publicadas neste ano e cujos nomes vêm abaixo em ordem alfabética.

Adriana Coser

Adriana Kelly Santos

Adriana Leão

Aisllan Asis

Alan Silva

Alberto de Araujo

Alexander Ramalho

Aline Cavaca

Alzira de Oliveria Jorge

Amanda Ornela

Ana Carolina Feldenheimer

Ana Luiza Ferrer

Ana María Rico

Ana Paula Melo

Ana Paula Remmi

Ana Silva

André Neves

Anneliese Wysocki

\author{
Beatriz Chagas \\ Berenice Temoteo da Silva \\ Bianca Leandro \\ Bianca Sophia \\ Catarina Resende \\ Cecília Soares \\ Charles Tesser \\ Cinira Magali Fortuna \\ Claudia Kessler \\ Claudia Miliauskas \\ Cristiane Silvestre de Paula \\ Cristiane Simôes \\ Daniel Vazquez \\ Daniela Biscarde \\ Edna Aparecida de Castro \\ Eliane Mazzuco dos Santos \\ Emanuele Marques \\ Erika Tomaz
}


Fabiana Pires

Fatima Cecchetto

Fatima Roquete

Fernanda Loureiro

Fernando Carneiro

Flávia Araújo

Flavia Freire

Fran Demétrio

Francini Guizardi

Frederico Policarpo

Helena Maria David

Helvécio Magalhães Júnior

Jane Sayd

João Cavalcante

João Marcos Bittencourt

José Ricardo Ayres

Juliana Rodrigues

Julyanne Felipette Lima

Kalline de Souza

Karla Giacomin

Kênia Silva

Laís Barbosa

Larissa Hermes Thomas Tombini

Laura Murray

Laura Sacchetti

Leila Maia

Lenita Claro

Liliana Angel

Lucas Tramontano

Luciana Caliman

Luciana Oliveira

Luciana Surjus

Luciane Guerra

Luiz Augusto Souza

Luiz Fernando Chazan

Luiza Costa
Manuela Muller

Marcia Agostini

Márcia Alves

Marcia Barçante Ladvocat

Marcilio Dantas Brandão

Marcos Senna

Marcos Vinicius Soares Pedrosa

Maria Claudia Carvalho

Maria Cristina Couto

Maria Cristina Ribeiro Furlan

Maria Eduarda Moraes

Maria Inês Moreira

Maria Lúcia Bosi

Maria Marta Amorim

Maria Stella Castro Lobo

Mariana Bteshe

Mariana Setubal

Marina Peduzzi

Mário Pereira Borba

Mayla Monteiro

Michele Cecille

Mirella Alves de Brito

Murilo Galvão Amancio Cruz

Nazareth Salutto

Neuma Chaveiro

Pablo Rocon

Patrícia Araújo

Patrícia Tavares Ribeiro

Patty Fidélis

Paula Cerqueira

Paula Gaudenzi

Paula Land

Paulo Fagundes

Rafael Agostini

Renarde Freire Nobre

Rodrigo Toniol 
Rogerio Azize

Ronaldo Teodoro

Rosa Rocha

Sabrina Calil-Elias

Sandra Fagundes

Sara Sousa

Shirley Prado

Silva Jardim

Sílvia Lüdorf

Suelen Rocha

Suzana Canez
Taisa Cortes

Thiago Pinheiro

Valéria Romano

Valeska Zanello

Vanessa Costa

Vanessa Rangel

Vivian Pizzinga

Wagner Camargo

Washington Castilhos

Wecisley Ribeiro Espírito Santo

Yana Tamara Tomasi 


\section{ERRATA}

No Editorial "Dois anos de pandemia: um balanço", com número de DOI: http:// dx.doi.org/10.1590/S0103-73312021310400 publicado no periódico Physis: Revista de Saúde Coletiva, v. 31, n. 4, e310400, na página 1, erro do email do autor:

ONDE SE LÊ:

Instituto de Medicina Social - Universidade do Estado do Rio de Janeiro, Rio de Janeiro-RJ, Brasil (dalpoz@uerj.br). ORCID: 0000-0002-3005-3280.

\section{LEIA-SE:}

Instituto de Medicina Social - Universidade do Estado do Rio de Janeiro, Rio de Janeiro-RJ, Brasil (dalpozm@uerj.br). ORCID: 0000-0002-3005-3280. 